\title{
INCIDÊNCIA DE COMPLICAÇÕES VASCULARES E CIRÚRGICAS EM TRANSPLANTE RENAL ENTRE 2013 E 2014 NA SANTA CASA DE MISERICÓRDIA DE GOIÂNIA
}

\author{
Incidence of vascular and surgical complications in renal transplants between 2013 and 2014 \\ at Santa Casa de Misericordia de Goiania
}

Ana Paula da Silva Azevedo Nora Bezerra, Fernanda Grazielle da Silva Azevedo Nora, Leandro Ferro de Moraes Bezerra, Fatima Mrue, Paulo Roberto de Melo Reis

\section{RESUMO}

Introdução: Embora o transplante renal represente uma perspectiva ao indivíduo portador de doença renal crônica terminal por se correlacionar a melhores índices de qualidade de vida e de morbimortalidade, esse procedimento não é isento de riscos. As taxas de complicações vasculares variam de $1-23 \%$ em todo mundo e guardam importância por estarem associadas a elevado risco de perda do enxerto. Objetivo: Avaliar a incidência de complicação vascular em pacientes submetidos a transplante renal na Santa Casa de Misericórdia de Goiânia, no período entre janeiro de 2013 a dezembro de 2014. Material e Métodos: Foram analisadas, através de 35 prontuários, as seguintes variáveis: estenose de artéria renal, trombose de artéria renal, estenose de veia renal, trombose de veia renal, pseudoaneurisma de artéria renal, fístula arteriovenosa, kinking de artéria renal, torção de enxerto e infarto. Foi coletado em prontuário: rim transplantado, tipo de doador, idade do receptor, gênero do receptor, reinternação e tempo de isquemia fria. Resultados: A população estudada incluiu 32 pacientes, sendo $34,38 \%$ do sexo feminino e $65,62 \%$ do sexo masculino, com média de idade de 46 anos. Entre as complicações cirúrgicas, ocorreram três casos de fistula urinária $(9,3 \%)$, dois casos de coleção $(6,25 \%)$, um caso de torção de enxerto $(3,12 \%)$ e um de estenose arterial $(3,12 \%)$. Todos os enxertos $(100 \%)$ foram de doador falecido e não houve perda de enxerto em nenhum caso $(0 \%)$. Conclusões: Embora o presente estudo tenha observado baixa incidência de complicação vascular relacionada a transplante renal, o TIF superior a $24 \mathrm{~h}$ foi o único fator de risco independente associado a tal evento.

Descritores: Doenças Vasculares; Transplante Renal; Doença Renal Crônica.

Instituições:

${ }^{1}$ Mestrado em Ciências Ambientais e Saúde, Pontifícia Universidade Católica de Goiás, Goiânia, Goiás, Brasil

${ }^{2}$ Laboratório de Bioengenharia e Biomecânica, Universidade Federal de Goiás, Goiânia, Goiás, Brasil

${ }^{3}$ Departamento de Medicina, Pontifícia Universidade Católica de Goiás, Goiânia, Goiás, Brasil.

\section{Correspondência:}

Ana Paula da Silva Azevedo Nora Bezerra

Rua T-38, n.1069, ap. 502, Ed. Bella Doro, Setor Bueno, Goiânia/GO Tel.: (62) 3521-1141

Email: anapnora@uol.com.br

Recebido em: 02/09/2015

Aceito em: 30/09/2015

\section{INTRODUÇÃO}

O número de indivíduos portadores de doença renal crônica (DRC) em estágio terminal, com necessidade de terapia renal substitutiva, vem aumentando gradativamente a cada ano. O aumento dessa incidência pode estar associado a fatores como o aumento na incidência de doenças que culminam com DRC, como diabetes mellitus e hipertensão arterial e melhoria no acesso aos meios diagnósticos e métodos de terapia substitutiva. ${ }^{1}$

Dentre as modalidades de terapia renal substitutiva, o transplante renal parece estar associado à melhor qualidade de vida do paciente portador de DRC em estágio terminal, mas essa terapia ainda se encontra em expansão pelo mundo. Foi inicialmente descrita em 
1951, na França, ${ }^{2}$ com resultados encorajadores, a partir de 1954, em Boston. O transplante renal foi consolidado na década de 1980, com a introdução da ciclosporina, elevando os dados de sobrevida do indivíduo para $90 \%$ e do enxerto para $80 \% .^{3}$

Os países com maior número de transplante renal são os Estados Unidos da América (EUA), China, Brasil e Índia. Os países com o melhor acesso da população ao transplante renal são Áustria, EUA, Croácia, Noruega, Portugal e Espanha3. Segundo a Organização Mundial de Saúde (OMS), os países com baixo índice de IDH (Índice de desenvolvimento Humano) apresentam número baixo de transplante renal. 4

Segundo o Registro Brasileiro de Transplantes, no ano de 2014, o transplante renal cresceu apenas $3,5 \%$, com aumento de $4,7 \%$ no número de transplantes com doador falecido e estabilização dos transplantes com doador vivo. O mesmo informativo coloca o Brasil como o segundo país em número absoluto de transplante renal. ${ }^{4}$

No ano de 2014, foram realizados 5.639 transplantes renais no Brasil, sendo 4.255 procedimentos relacionados a doadores falecidos e 1.384 a doadores vivos, gerando um índice de 29,6 transplantes renais pmp (por milhão de população). No estado de Goiás, no ano de 2014, foram realizados 65 transplantes renais, sendo 22 com doadores vivos e 43 com doadores falecidos, com índice de 10,8 pmp. ${ }^{4}$

No transplante renal, a sobrevida do paciente e do enxerto varia de acordo com o tipo do doador. Quando o enxerto é proveniente de doador vivo, obtém-se uma sobrevida média no primeiro ano de $95 \%$ para o receptor e $90 \%$ para o enxerto. Quando o enxerto é proveniente de doador falecido, a sobrevida média no primeiro ano é de $88 \%$ para o receptor e $78 \%$ para o enxerto. ${ }^{4}$

Até dezembro de 2014 , havia 18.147 pacientes ativos na lista de espera para um transplante renal no Brasil e, no estado de Goiás, essa lista contava com 332 pacientes ativos no mesmo período. ${ }^{4}$ Segundo a Central de Transplantes do Estado de Goiás, atualmente estão registradas três instituições para a realização de transplante renal no estado que são Santa Casa de Misericórdia de Goiânia, Hospital Santa Genoveva e Hospital Geral de Goiânia Dr. Alberto Rassi.

O transplante renal representa uma perspectiva ao indivíduo portador de doença renal crônica terminal, em termos de qualidade de vida e de morbimortalidade. Por outro lado, esse procedimento não é isento de riscos. A evolução na técnica do transplante renal visa diminuir esses riscos para os indivíduos submetidos a essa terapia, no entanto, complicações associadas ao procedimento cirúrgico podem ocorrer.
O objetivo do presente estudo foi avaliar a incidência de complicação vascular nos pacientes submetidos a transplante renal na Santa Casa de Misericórdia de Goiânia, no período entre janeiro de 2013 e dezembro de 2014, através da análise dos respectivos prontuários, tendo sido escolhida essa instituição devido à maior organização e fidelidade de dados em seus prontuários.

\section{MÉTODOS}

Estudo retrospectivo, descritivo, analítico, envolvendo análise de prontuários de pacientes submetidos a transplante renal no Serviço de Urologia da Santa Casa de Misericórdia de Goiânia e seguidos pelo Serviço de Nefrologia da mesma instituição.

Foram analisados 35 prontuários de pacientes submetidos à cirurgia de transplante renal pelo serviço de urologia da Santa Casa de Misericórdia de Goiânia e acompanhados pelo serviço de Nefrologia da mesma instituição, no período de janeiro de 2013 a dezembro de 2014.

O período do estudo foi estabelecido entre janeiro de 2013 e dezembro de 2014 pela disponibilidade de prontuário médico para análise. Prontuários anteriores a esse período não se encontravam disponíveis para análise e coleta de dados.

\section{CRITÉRIOS DE INCLUSÃO}

Pacientes submetidos a transplante renal na Santa Casa de Misericórdia de Goiânia no período de janeiro de 2013 a dezembro de 2014.

\section{CRITÉRIOS DE EXCLUSÃO}

Pacientes submetidos a transplante renal associado a qualquer outro procedimento cirúrgico em um mesmo tempo (por exemplo, transplante rim-pâncreas).

Pacientes em que o tempo de isquemia fria (TIF) não estava disponível para coleta em prontuário.

\section{COLETA DE DADOS}

As variáveis analisadas em prontuário médico foram: estenose de artéria renal, trombose de artéria renal, estenose de veia renal, trombose de veia renal, pseudoaneurisma de artéria renal, fístula arteriovenosa, kinking de artéria renal, torção de enxerto e infarto.

Os dados coletados em prontuário médico foram: rim transplantado (direito $\mathrm{x}$ esquerdo), tipo de doador (vivo $x$ falecido), idade do receptor, gênero do receptor (masculino $x$ feminino), outras complicações (infecção e outras complicações cirúrgicas), reinternação (causas) e tempo de isquemia fria (TIF). 
Ana Paula da Silva Azevedo Nora Bezerra, Fernanda Grazielle da Silva Azevedo Nora, Leandro Ferro de Moraes Bezerra, Fatima Mrue, Paulo Roberto de Melo Reis

\section{ANÁLISE ESTATÍSTICA}

A análise estatística foi realizada pelo software Statistica 10.0 (Dell). Após verificação da normalidade das distribuições e da homogeneidade dos dados por meio do teste de Chi-Quadrado, em que se obteve um $p=0,197$; foi aplicado teste de normalidade de Kolmogorov-Smirnov, por não apresentar distribuição normal. Foram aplicados testes não paramétricos de Kruskall-Wallis com pos-hoc de Tukey a fim de verificar diferenças entre as variáveis analisadas, utilizando como referência estatística o nível de significância de $5 \%(p \leq 0,05)$. As variáveis são apresentadas percentual.

\section{ASPECTOS ÉTICOS}

O presente estudo foi avaliado e aprovado pelo Comitê de Ética em Pesquisa da Santa Casa de Misericórdia de Goiânia. Aprovado sob o protocolo de número 021086/2015.

\section{RESULTADOS}

Foram analisados 35 prontuários de pacientes submetidos a cirurgia de transplante renal pelo serviço de urologia da Santa Casa de Misericórdia de Goiânia e acompanhados pelo serviço de Nefrologia da mesma instituição, no período de janeiro de 2013 a dezembro de 2014.

Desses 35 prontuários, três pacientes foram excluídos por não ter TIF identificado em seus prontuários (critério de exclusão). Entre os 32 pacientes que cumpriam os critérios de inclusão, 21 (65,62\%) eram do sexo masculino e 11 (34,38\%), do sexo feminino, com idade entre 17 e 66 anos (média: 46 anos), como é mostrado na tabela 1.

Tabela 1 - Distribuição dos receptores, de acordo com o gênero e idade

\begin{tabular}{lcc}
\hline \multicolumn{1}{c}{ Receptor } & Número & Porcentagem \\
\hline Masculino & 21 & $65,62 \%$ \\
Feminino & 11 & $34,38 \%$ \\
Idade & 46,43 anos (média) & \\
\hline
\end{tabular}

Os $32(100 \%)$ pacientes receberam enxerto renal de doador falecido. O tempo de isquemia fria (TIF) variou entre 14 e 42 horas, com média de 23,03 horas; sendo que em três casos não houve relato do tempo de isquemia fria, ocorrendo, então, a exclusão destes do estudo. Em 17 (53,12\%) casos houve reinternação após o transplante renal, ao longo do primeiro ano, sendo que
$16(94,11 \%)$ foi por infecção e uma $(5,89 \%)$ ocorreu para pulsoterapia por piora da função renal do enxerto.

De acordo com os prontuários analisados, todos os 32 $(100 \%)$ casos foram submetidos a anastomose arterial término-lateral, sendo seis $(18,75 \%)$ casos em artéria ilíaca comum direita, um $(3,12 \%)$ em artéria ilíaca interna direita, 19 (59,37\%) em artéria ilíaca externa, sendo 17 à direita e 2 à esquerda. Em seis $(18,75 \%)$ casos, não foram encontrados registros de qual artéria foi utilizada para anastomose.

Quanto ao enxerto utilizado, a tabela 2 mostra que, 15 $(46,87 \%)$ pacientes receberam o rim direito do doador, oito $(25 \%)$ receberam o rim esquerdo e nove $(28,12 \%)$ não tiveram registro do rim transplantado.

Tabela 2 - Características dos transplantes renais realizados

\begin{tabular}{llcc}
\hline \multicolumn{1}{c}{ Transplante } & & Número & Porcentagem \\
Doador & Falecido & 32 & $100 \%$ \\
& Vivo & 0 & 0 \\
Reinternação & & 17 & \\
& Infecção & 16 & $94,11 \%$ \\
& Pulsoterapia & 01 & $5,89 \%$ \\
TIF & & & \\
& & $23,03 \mathrm{~h}$ (média) & \\
Anastomose & Artéria ilíaca & 06 & $18,75 \%$ \\
arterial & comum & & $3,12 \%$ \\
& $\begin{array}{l}\text { Artéria ilíaca } \\
\text { comum }\end{array}$ & 01 & $59,37 \%$ \\
& Artéria ilíaca & 19 & $18,75 \%$ \\
\hline & comum & & \\
& Não registrado & 06 & \\
\hline
\end{tabular}

Foram registrados sete $(21,87 \%)$ casos com complicações cirúrgicas, sendo três $(9,3 \%)$ casos de fístula urinária, dois $(6,25 \%)$ de coleção, um $(3,12 \%)$ de torção de enxerto e um $(3,12 \%)$ de estenose arterial. Não houve perda de enxerto $(0 \%)$ no período analisado. As complicações vasculares encontradas foram: um caso de estenose arterial e um de torção de enxerto, com um total de dois $(6,25 \%)$.

Os casos que apresentaram fístula urinária (três casos) foram submetidos a correção cirúrgica em até 30 dias após o transplante. Os dois casos de coleção foram drenados e submetidos à antibioticoterapia na mesma internação que a do transplante renal, sem perda de enxerto e/ou da função renal.

O paciente que apresentou torção de enxertia (um caso) era do sexo masculino, com 53 anos ao ser submetido ao transplante renal, tendo recebido o rim direito de doador falecido, realizada anastomose término-lateral com artéria ilíaca comum direita com patch (artéria curta 
do enxerto) e apresentado TIF de 16 horas. Ao mesmo tempo, o paciente também apresentou fistula urinária, reoperado no $7^{\circ}$ dia após o transplante, para ressutura de parede (correção de deiscência), correção de fístula urinária e correção da torção arterial do enxerto, com reimplante do mesmo, sem a perda do enxerto.

Tabela 3 - Complicações cirúrgicas relacionadas aos transplantes renais

\begin{tabular}{lcc}
\hline Complicação Cirúrgica & Número & Porcentagem \\
\hline Fístula urinária & 03 & $9,3 \%$ \\
Coleção & 02 & $6,25 \%$ \\
Complicação vascular & 02 & $6,25 \%$ \\
Total & 07 & $21,87 \%$ \\
\hline
\end{tabular}

O paciente que apresentou estenose arterial de $60 \%$ de artéria do rim transplantado foi diagnosticado em doppler de rotina, três meses após o transplante, sem perda de função renal ou alteração de resistência vascular renal. Esse paciente é do sexo masculino, tinha 59 anos ao realizar o transplante renal, recebeu o rim direito de doador falecido, apresentou tempo de isquemia fria de 26 horas. Ainda sem programação de intervenção.

Tabela 4 - Complicações vasculares, características do receptor e do transplante renal

\begin{tabular}{lcccc}
\hline $\begin{array}{c}\text { Complicação } \\
\text { vascular }\end{array}$ & Gênero & Idade & $\begin{array}{c}\text { Rim } \\
\text { transplantado }\end{array}$ & TIF \\
\hline Torção & Masculino & 53 anos & Direito & $16 \mathrm{~h}$ \\
Estenose & Masculino & 59 anos & Direito & $26 \mathrm{~h}$ \\
arterial & $\mathrm{P}=0.06$ & $\mathrm{P}=0.3$ & $\mathrm{P}=0.67$ & $\mathrm{P}=0.034$ \\
& & &
\end{tabular}

Quando analisamos o TIF menor de $24 \mathrm{hs}$ com o maior de $24 \mathrm{hs}$, percebemos que não houve diferença estatisticamente significativa, apresentando o valor de $p=0,067$. Por outro lado, quando cruzamos a variável TIF com complicações vasculares, percebemos que quando o TIF é maior que $24 \mathrm{hs}$, apresenta diferença estatisticamente significativa quando relacionado com complicações vasculares, apresentando o $p=0,034$. Já o TIF menor que $24 \mathrm{hs}$ não apresenta diferença estatisticamente significativa quando comparado com complicações vasculares, apresentando o valor de $p=0,058$.

Ao se comparar o TIF menor de $24 \mathrm{hs} \mathrm{com} \mathrm{o} \mathrm{maior} \mathrm{de}$ $24 \mathrm{hs}$, apresentado na tabela 4 percebe-se que não há diferença estatisticamente significativa $(p=0,067)$. Por outro lado, ao avaliar a variável TIF com complicações vasculares, verifica-se que quando o TIF é maior que 24hs, há diferença estatisticamente significativa, com complicações vasculares $(p=0,034)$. Já o TIF menor que $24 \mathrm{hs}$ não apresenta diferença estatisticamente significativa quando comparado com complicações vasculares $(p=0,058)$. No entanto, não houve diferença estatística significativa $(p<0,05)$ entre lesão vascular apresentada e rim transplantado, gênero e idade.

No período analisado, foram registrados dois $(6,25 \%)$ óbitos de pacientes submetidos a transplante renal. Ambos apresentaram sepse de foco pulmonar com óbito em período superior a seis meses, após o transplante renal.

\section{DISCUSSÃO}

Em 2014, o estado de Goiás apresentava uma população de 6.003.788 habitantes, segundo o IBGE, com necessidade estimada de 360 transplantes de rim (acometendo 0,005\% da população do Estado), mas com realização de 65 (18\%). Desses 65 transplantes renais realizados, 22 (33\%) foram com doador vivo e 43 (66\%) com doador falecido, enquanto que, a nível nacional, foram realizados 5639 transplantes renais, sendo 4255 de doador falecido ( $75 \%$ dos casos). ${ }^{4}$ Embora o estado de Goiás possua o equivalente a $3,14 \%$ da população do país, o total de transplantes realizados representou $1,15 \%$ em relação ao total nacional. A grande dificuldade em se aumentar o número de potenciais doadores falecidos no estado, está relacionada à recusa familiar com relação á doação de órgãos. ${ }^{4}$

Em uma análise de três décadas de transplante renal, Bessed et al. (2012) verificaram que, em 3129 transplantes renais, houve 421 eventos vasculares $(13,5 \%)$, sendo a estenose arterial do enxerto transplantado o mais comum (78\%). Nesse mesmo estudo, verificou-se que a ocorrência de evento vascular leva a um decréscimo de três anos na sobrevida do enxerto. Portanto, para diminuir a incidência de perda de atividade do enxerto relacionado à lesão vascular, deve-se buscar treinamento constante dos cirurgiões envolvidos no transplante, evitar a tração dos vasos sanguíneos durante a captação do órgão, seleção adequada do doador e do receptor, veia renal direita com extensão e anastomose término-lateral dos vasos. ${ }^{5}$

O doador vivo com múltiplas artérias não poderia ser considerado doador potencial, devido ao maior risco associado à disfunção do enxerto, hipertensão arterial, maior incidência de complicações urológicas e vasculares. ${ }^{6} \mathrm{O}$ principal risco associado é de estenose de artéria renal, em que Emiroglu et al. (2000) encontraram uma incidência de 4,1\%, na literatura no geral (3-23\%). 
Ana Paula da Silva Azevedo Nora Bezerra, Fernanda Grazielle da Silva Azevedo Nora, Leandro Ferro de Moraes Bezerra, Fatima Mrue, Paulo Roberto de Melo Reis

Portanto, tanto o doador vivo quanto o falecido com múltiplas artérias renais podem ser doadores efetivos, pois não se observou alteração na sobrevida do enxerto de acordo com o número de artérias envolvidas, aumentando assim o número de doadores efetivos. ${ }^{7}$

A estenose de artéria renal possui causa multifatorial, associada principalmente à falha na técnica cirúrgica, tipo de doador, fatores imunológicos e infecção viral. Durante o procedimento cirúrgico, pode ocorrer trauma nos vasos do enxerto (manipulação, clampeamento ou sutura dos mesmos), gerando um quadro de estenose precoce. Tempo de isquemia fria prolongada também pode levar a um quadro de lesão endotelial com estenose secundária. ${ }^{6}$ No único caso de estenose arterial encontrado entre os 32 transplantes estudados, o paciente apresentou quadro de estenose precoce (três meses), com tempo de isquemia prolongada (26 horas), sem, no entanto, desenvolver o quadro clínico de hipertensão arterial e/ou disfunção renal. ${ }^{8}$

Tempo de isquemia fria é o período entre a perfusão com a solução de preservação e o restabalecimento do fluxo sanguineo no receptor. Durante o TIF, ocorre a lesão celular através da inibição da bomba de sódio, troca do cloro extracelular pelo potássio intracelular, acúmulo de água intracelular, edema celular, consumo de reservas energéticas celulares com formação de radicais livres. ${ }^{9}$ Portanto, quanto mais breve for esse período, menores serão os danos celulares do enxerto. É considerado tempo de isquemia fria prolongada quando esse período ultrapassa 24hs (quando a solução de preservação é a Euro-Collins) no transplante renal. ${ }^{10}$

No presente estudo, o paciente que desenvolveu estenose de artéria renal apresentou TIF de 26hs, sendo, portanto, prolongado e funcionando como uma causa predisponente para a estenose. $6 \mathrm{De}$ acordo com a significância estatística encontrada entre complicações vasculares e TIF superior a 24hs $(p=0,034)$, pode-se inferir que o TIF superior a $24 \mathrm{hs}$ apresenta-se como fator de risco para complicação vascular.

A estenose de artéria renal possui incidência variável, em torno de 1-23\%. O diagnóstico geralmente é realizado por ultrassonografia-doppler, e, quando há disfunção renal, o tratamento de eleição é a angioplastia percutânea, com índice de sucesso de 60-94\% dos casos. ${ }^{11}$ O presente estudo apresentou uma taxa de estenose de artéria renal de 3,12\%, valor semelhante ao apresentado na literatura. $O$ diagnóstico foi realizado por ultrassonografia-doppler de rotina aos três meses, mesmo sem o paciente apresentar disfunção renal.

Para o rastreamento de estenose de artéria renal, vários exames de imagem podem ser utilizados. A angiotomografia e a angiorressonância são exames de alta acurácia, mas de custo elevado e com risco ao enxerto pelo uso de contraste. A angiotomografia tem risco adicional ao uso de radiação. A angiorressonância está associada à superestimação do grau de estenose de um vaso, mas é o método de escolha quando há a limitação à utilização da ultrassonografia-doppler. ${ }^{12,13}$

A ultrassonografia-doppler é o método não invasivo de imagem de escolha para rastreamento de estenose de artéria renal, por apresentar boa acurácia e ser isento de riscos. Apresenta como desvantagem o fato de ser operador-dependente e aparelho-dependente. ${ }^{14,15}$

Para que a estenose de artéria renal seja considerada hemodinamicamente significativa à ultrassonografiadoppler (estenose > 50-60\%), deve-se demonstrar os seguintes critérios: pico de velocidade sistólica ao nível da anastomose ou ao longo da artéria renal superior a $200-300 \mathrm{~cm} / \mathrm{s}$; índice ilíaco-renal (relação entre os picos de velocidade sistólica da artéria renal e artéria ilíaca externa) igual ou superior a 2,0; tempo de aceleração superior a $100 \mathrm{~ms}$ na região hilar. ${ }^{16}$ No presente estudo, houve o diagnóstico de um caso de estenose de artéria renal de $60 \%$ a ultrassonografia-doppler sem alteração da resistência vascular.

A torção do enxerto renal é uma condição rara que leva a um evento final de infarto do parênquima renal, com disfunção ou perda renal. Os sintomas apresentados são inespecíficos: disfunção renal, dor abdominal, oligúria ou anúria, náuseas e vômitos, febre, diarréia, ganho de peso e edema. Esses sintomas geram uma confirmação diagnóstica tardia, com retardo na intervenção terapêutica eficaz. Quando submetido à restauração do fluxo sanguíneo precoce, há salvamento do enxerto e de sua função, em $44 \%$ dos casos. ${ }^{17}$ No presente estudo, ocorreu um caso de torção de enxerto, com correção cirúrgica precoce e sem perda da função renal.

A principal causa de reinternação hospitalar nos primeiros seis meses após o transplante renal é a infecção, principalmente no primeiro mês póstransplante renal (50\% dos casos), sendo uma das principais causas de óbito em paciente pós-transplante nos primeiros seis meses. ${ }^{18,19}$ Os fatores de risco para rehospitalização são: doador falecido, uso de imunossupressor e infecção por CMV. ${ }^{20} \mathrm{Em}$ um estudo que analisou 419 transplantes renais, encontraram $45,5 \%$ de reinternações hospitalares, por infecção. No presente estudo, foram registrados 17 (53,12\%) casos de reinternação hospitalar no primeiro ano após transplante renal, sendo $16(94,11 \%)$ casos por infecção.

Um estudo encontrou $84 \%$ de reinternação nos primeiros seis meses pós-transplante renal, associadas a complicação cirúrgica. ${ }^{21}$ Outro estudo encontrou que as reinternações associadas a complicações cirúrgicas, 
pois estas foram corrigidas durante a primeira internação (para a realização do transplante renal). ${ }^{22}$ O presente estudo verificou um índice de $21,87 \%$ (sete casos) de complicação cirúrgica, no entanto seis casos tiveram a correção da complicação cirúrgica na mesma internação hospitalar do transplante renal, e apenas um caso (estenose arterial) foi verificado no terceiro mês de acompanhamento ambulatorial.

A taxa de complicação urológica em transplante renal varia de 4 a $20 \%$, de acordo com a literatura, e pode estar associado à falha técnica, isquemia ou infecção. ${ }^{23}$ A complicação urológica encontrada nos pacientes analisados foi a fístula urinária, com um índice de 9,3\%.

Por se tratar de um evento de baixa incidência, a análise das complicações vasculares relacionadas ao transplante renal deve ser realizado com o maior número possivel de indivíduos. No presente estudo, o número de individuos avaliados foi baixo; por isso, as demais complicações vasculares, tais como: trombose arterial, estenose de veia renal, trombose de veia renal, pseudoaneurisma, kinking e infarto, não foram encontradas.
As possíveis associações das complicações vasculares no transplante renal, com características do receptor e do doador, foram prejudicadas pela baixa ocorrência do evento e por informações incompletas presentes em prontuários médicos.

\section{CONCLUSÃO}

O presente estudo apresentou baixa incidência de complicação vascular relacionada ao transplante renal. Foram observados dois eventos de complicação vascular nos casos analisados, estando associados à estenose de artéria renal e o outro associado à torção de enxerto. No presente estudo, o TIF superior a 24 horas foi o fator de risco associado à complicação vascular em transplante renal.

Não foi verificada associação entre complicação vascular e rim transplantado, tipo de doador, idade e gênero do receptor.

\section{ABSTRACT}

Introduction: Although renal transplantation represents a perspective for individuals with terminal chronic kidney disease, as it is correlated to better quality of life, the morbidity and mortality rates, this procedure is not risk free. The rates of vascular complications vary from $1-23 \%$ worldwide, and they are important for being associated with high risk of graft loss. Purpose: To assess the incidence of vascular complications in patients submitted to renal transplantation at Santa Casa de Misericórdia de Goiânia from January 2013 to December 2014. Material and Methods: The following variables were analyzed: renal artery stenosis, renal artery thrombosis, renal vein stenosis, renal vein thrombosis, renal artery pseudoaneurysm, arteriovenous fistula, renal artery kinking, graft twist and infarction. Data collected from medical records: transplanted kidney, donor type, recipient age, recipient gender, rehospitalization and time of cold ischemia. Results: The studied population included 32 patients, being $34.38 \%$ female and $65.62 \%$ male, with mean age of 46 years. Among surgical complications, there were three cases of urinary fistula (9.3\%), two cases of collection $(6.25 \%)$, one case of graft twist (3.12\%) and one case of arterial stenosis (3.12 \%). All grafts (100\%) came from deceased donor, and there was no graft loss in any case (0\%). Conclusion: Although the present study observed a low incidence of vascular complications related to renal transplantation, TIF over 24 hours was the only independent risk factor associated with such event.

Keywords: Vascular diseases; Kidney Transplantation; Renal Insufficiency, Chronic. 
Ana Paula da Silva Azevedo Nora Bezerra, Fernanda Grazielle da Silva Azevedo Nora, Leandro Ferro de Moraes Bezerra, Fatima Mrue, Paulo Roberto de Melo Reis

\section{REFERÊNCIAS}

1. Mezzomo N, Pansard H, Arantes L, Rempel W, Argenta L, Rodrigues A et al. Sobrevida em hemodiálise crônica: estudo de uma coorte de 1.009 pacientes em 25 anos. Jornal Brasileiro de Nefrologia. 2009;31(3):190-7.

2. Kuss R, Teinturier J, Milliez P. Some attempts at kidney trasplantation in man. Mem Acad Chir (Paris); p. 77:755. 1951.

3. Garcia G, Harden P, Chapman J. O papel global do transplante renal. Jornal Brasileiro de Nefrologia. 2012;34(1):01-07.

4. Dimensionamento dos transplantes no Brasil e em cada estado (2007-2014) [Internet]. Registro Brasileiro de Transplantes, Ano XX número 4, 2014. 2017 [cited 15 March 2016]. Available from:

http://www.abto.org.br/abtov03/Upload/file/RBT/2014/rbt2014parcial(1).pdf

5. Bessede T, Droupy S, Hammoudi Y, Bedretdinova D, Durrbach A, Charpentier B et al. Surgical prevention and management of vascular complications of kidney transplantation. Transplant International. 2012;25(9):994-1001.

6. Fernández-Nájera J, Beltrán S, Aparicio M, Molina P, Gavela E, Ávila A et al. Transplant Renal Artery Stenosis: Association With Acute Vascular Rejection. Transplantation Proceedings. 2006;38(8):2404-5.

7. Emiroğlu R, Köseoğlu F, Karakayalı H, Bilgin N, Haberal M. Multiple-artery anastomosis in kidney transplantation. Transplantation Proceedings. 2000;32(3):617-9.

8. Dickerman R, Peters P, Hull A, Curry T, Atkins C, Fry W. Surgical Correction of Posttransplant Renovascular Hypertension. Annals of Surgery. 1980;192(5):639-44.

9. Belzer F, Southard J. Principles of solid-organ preservation by cold storage. Transplantation. 1988;45(4):673-6.

10. Clavien P, Harvey P, Strasberg S. Preservation and reperfusion injuries in liver allografts. Transplantation. 1992;53(5):957-78.

11. Ferraz Neto B, Thomé T, Moura L. Curso Prático de extração, perfusão e acondicionamento de múltiplos orgãos para Transplante. Hospital Israelita Albert Einstein. 2012;1(2012).

12. Dimitroulis D, Bokos J, Zavos G, Nikiteas N, Karidis $\mathrm{N}$, Katsaronis $\mathrm{P}$ et al. Vascular Complications in Renal Transplantation: A Single-Center Experience in 1367 Renal Transplantations and Review of the Literature. Transplantation Proceedings. 2009;41(5):1609-14.
13. Aktas S, Boyvat F, Sevmis S, Moray G, Karakayali H, Haberal M. Analysis of Vascular Complications After Renal Transplantation. Transplantation Proceedings. 2011;43(2):55761.

14. Cianci R, Coen G, Mandredini P, Ciano G, Di Donato $D$, Stivali G. Diagnosis and Outcome of Renal Function in Patients With Renal Artery Stenosis: Which Role Have Color Doppler Sonography and Magnetic Resonance Angiography?. Minerva Cardioangiol. 2006;54:139-44.

15. Zierler R. Is duplex scanning the best screening test for renal artery stenosis?. Seminars in Vascular Surgery. 2001;14(3):177-185.

16. PAVEN G, WAUGH R, NICHOLSON J, GILLIN A, HENNESSY A. Screening tests for renal artery stenosis: A case-series from an Australian tertiary referral centre. Nephrology. 2006;11(1):68-72.

17. Krumme B. Renal Doppler Sonography - Update in Clinical Nephrology. Nephron Clinical Practice. 2006;103(2):c24-c28.

18. Maffei F, Lastória S, Bonetti Yoshida W. Doenças vasculares periféricas (4a. ed.). 1st ed. Grupo Gen - Guanabara Koogan; 2000.

19. Lucewicz A, Isaacs A, Allen R, Lam V, Angelides S, Pleass $\mathrm{H}$. Torsion of intraperitoneal kidney transplant. ANZ Journal of Surgery. 2011;82(5):299-302.

20. Boubaker K, Harzallah A, Ounissi M, Becha M, Guergueh T, Hedri $\mathrm{H}$ et al. Rehospitalization After Kidney Transplantation During the First Year: Length, Causes and Relationship With Long-term Patient and Graft Survival. Transplantation Proceedings. 2011;43(5):1742-6.

21. Neylan J, Sullivan E, Steinwald B, Goss T. Assessment of the frequency and costs of posttransplantation hospitalizations in patients receiving tacrolimus versus cyclosporine. American Journal of Kidney Diseases. 1998;32(5):770-777.

22. Charfeddine K, Zaghden S, Kharrat M, Kamoun K, Jarraya F, Hachicha J. Infectious Complications in Kidney Transplant Recipients: A Single-Center Experience. Transplantation Proceedings. 2005;37(6):2823-2825.

23. Pisani F, laria G, D’Angelo M, Rascente M, Barletta A, Rizza $\checkmark$ et al. Urologic Complications in Kidney Transplantation. Transplantation Proceedings. 2005;37(6):2521-2. 